\title{
Localized geometric topologies with bounded node degree for three-dimensional wireless sensor networks
}

\author{
Fan $\mathrm{Li}^{1}$, Zeming Chen ${ }^{1}$ and $\mathrm{Yu} \mathrm{Wang}^{2^{*}}$
}

\begin{abstract}
Three-dimensional (3D) wireless sensor networks have attracted a lot of attention due to their great potential usages in both commercial and civilian applications, such as environmental data collection, pollution monitoring, space exploration, disaster prevention, and tactical surveillance. Topology control in 3D sensor networks has been studied recently, and different 3D geometric topologies were proposed to be the underlying network topologies to achieve the sparseness of the communication networks. However, most of these proposed 3D topologies cannot bound the maximum node degree, i.e., some nodes may need to maintain a large number of neighbors in the constructed topologies, which is not energy efficient and may lead to large contention. In this article, we extend several existing 3D geometric topologies to a set of new 3D topologies with bounded node degree. We provide both theoretical analysis and simulation evaluation on their power efficiency and node degree distributions. Our simulation results over random 3D sensor networks confirm nice performances of these proposed 3D topologies.
\end{abstract}

\section{Introduction}

Due to its wide-range potential applications (such as environmental data collection, pollution monitoring, space exploration, disaster prevention, and tactical surveillance), 3D wireless sensor network has recently emerged as a premier research topic. Most current researches in 3D sensor networks primarily focus on coverage [1-4], connectivity $[4,5]$, and routing issues [6-12]. In this article, we focus on how to efficiently control the 3D network topology to maintain both network connectivity and energy efficiency of routes in 3D wireless sensor networks.

Topology control [13-15] has been well-studied for 2D wireless ad hoc and sensor networks in the past decade [16-26]. Topology control methods allow each sensor node to locally adjust its transmission range and select certain neighbors for communication, while maintaining a structure that can support energy efficient routing and improve the overall network performance. Given the dynamic nature of sensor networks, the topology should

\footnotetext{
* Correspondence: yu.wang@uncc.edu

${ }^{2}$ Department of Computer Science, University of North Carolina at Charlotte, Charlotte, NC 28223, USA

Full list of author information is available at the end of the article
}

be locally and self-adaptively maintained without affecting the whole network and the communication cost during maintaining should not be too high. There exist several topology control techniques such as localized geometrical structures [17-25], dynamic cluster techniques [27-30] and power assignment protocols [31-37]. In this article, we focus on geometrical structures based methods.

Though many 2D geometrical structures have been proposed, surprisingly, there is not much study of 3D geometrical methods for topology control in 3D sensor networks, except for [38-44]. Bahramgiri et al. [38], Ghosh et al. [42], and Poduri et al. [43] proposed methods based on generalized cone-based topology control algorithm $[17,20]$ to preserve connectivity in $3 \mathrm{D}$ sensor networks, however, all of their 3D structures cannot bound the node degree, i.e., some nodes may have large unbounded number of neighbors. In addition, their construction methods are complex. Wang et al. [39-41] and Kim et al. [44] then proposed a set of 3D structures based on Yao structures $[18,19]$ in 3D space. These 3D Yao structures can be constructed locally and efficiently, and they do have bounded node out-degree. However, none of these existing 3D structures can bound the 
node in-degree. Possible unbounded in-degree at some nodes will often cause large overhead or contention at those nodes which may make them exhausted earlier than other nodes. Faced up with this challenge, in this article we study how to efficiently construct 3D topology with bounded node degree (both out-degree and in-degree) to maintain connectivity, conserve energy, and enable energy efficient routing. In particularly, we propose two general frameworks: 3D Symmetric Yao Graph and 3D Yao and Reverse Yao Graph, which are based on any existing 3D Yao structures. We then theoretically prove that the new 3D structures built by our proposed method can guarantee connectivity of the network, have a constant-bounded node degree, and support energy efficient routes. We believe that these structures are the first set of 3D structures that can achieve all of these properties.

The rest of this article is organized as follows. Section 2 introduces the network model and desired properties of our 3D topology control problem. Section 3 provides a brief review of related study on existing 3D topology control methods. Section 4 presents the algorithms to build 3D Yao-based topologies with bounded degree and the theoretical proofs of their nice properties, such as connectivity, degree bound, and power stretch factor. Section 5 presents the simulation results on random 3D networks and Section 6 concludes the article.

\section{Network models and assumptions}

A 3D wireless network consists of a set $V$ of $n$ wireless nodes distributed in a 3D plane $\mathbb{R}^{3}$. Each node has the same maximum transmission range $R$. These wireless nodes define a unit ball graph (UBG), or called unit sphere graph, in which there is an edge $u v$ between two nodes $u$ and $v$ iff (if and only if) the Euclidean distance II $u v \|$ between $u$ and $v$ in $\mathbb{R}^{3}$ is at most $R$. In other words, two nodes can always receive the signal from each other directly if the distance between them is not more than $R$. If there exists a link $u v$ in UBG, $v$ is a neighbor of $u$. All neighbors of $u$ form its one-hop neighborhood, denoted as $N_{U B G}(u)$ or $N(u)$. The size of $N_{U B G}(u)$ is the node degree of $u$. We assume that all wireless nodes have distinctive identities and each node knows its position information either through a low-power GPS receiver or some other ways (such as 3D localization methods in [45-47]). By one-hop broadcasting, each node $u$ can gather the location information of all nodes within its transmission range. As in the most common power-attenuation model, the power to support a link $u v$ is assumed to be $\|$ $u v \|\left.\right|^{\beta}$, where $\beta$ is a real constant between 2 and 5 depending on the wireless transmission environment.

Topology control protocols aim to maintain a structure $H$ from the original communication graph UBG that can preserve connectivity, optimize network throughput with power-efficient routing and conserve energy. The constructed topology $H$ could be a directed or undirected graph. In the literature, the following desirable features of the structure are well-regarded and preferred in wireless sensor networks:

(1) Connectivity: To guarantee communications among all sensor nodes, the constructed topology $H$ needs to be connected, i.e., there exists a path between any pair of nodes in the topology. This is the most fundamental requirement of topology control. Here, we always assume that the original communication graph UBG is a connected graph.

(2) Bounded node degree: It is also desirable that node degree in the constructed topology $H$ is small and upper-bounded by a constant. If $H$ is a directed graph, both in-degree and out-degree should be bounded. A small node degree reduces the MAC-level contention and interference, and may help to mitigate the wellknown hidden and exposed terminal problems. In addition, if a graph has a bounded node degree, it is also a sparse graph, i.e., the total number of links is linear with the total number of nodes in the graph. A sparse graph conserves more energy in term of maintaining the constructed network topology.

(3) Power spanner: A good network topology should be energy efficient, i.e., the total power consumption of the least energy cost path between any two nodes in final topology should not exceed a constant factor of the power consumption of the least energy cost path in original network [18]. Given a path $v_{1} v_{2} \cdots v_{h}$ connecting two nodes $v_{1}$ and $v_{h}$, the energy cost of this path is $\sum_{j=1}^{h-1}\left\|v_{j} v_{j+1}\right\|^{\beta}$. The path with the least energy cost is called the shortest path in a graph. A subgraph $H$ is called a power spanner of a graph $G$ if there is a positive real constant $\rho$ such that for any two nodes, the power consumption of the shortest path in $H$ is at most $\rho$ times of the power consumption of the shortest path in $G$. The constant $\rho$ is called the power stretch factor. A power spanner of the communication graph (e.g., UBG) is usually energy efficient for routing.

(4) Localized construction: Due to limited resources and high mobility of wireless nodes, it is preferred that the topology can be constructed locally and in a selforganizing fashion. Here, a topology is localized, i.e., can be constructed locally, if every node $u$ can decide all edges incident on itself in the topology by only using the information of nodes within a constant hops of $u$. Actually, all construction algorithms of our topologies presented here only use 1-hop neighbor information.

\section{Related works}

In this section, we briefly review the current techniques of topology control in both 2D and 3D wireless networks. 


\subsection{Topology control in 2D networks}

With the objective of achieving energy efficiency and maintaining network connectivity, several localized geometrical structures have been proposed for topology control in 2D wireless networks, such as local minimum spanning tree (LMST) [21,22], relative neighborhood graph (RNG) [48,49], Gabriel graph (GG) [48,50], Yao graph (YG) [18,19], cone-based topology control (CBTC) [17,20], Delaunay-based graph [23-25], and different combinations of these graphs [51-53]. By constructing such sparse topology structures, transmission power of nodes can be minimized. As a result, the number of links in the constructed topology is significantly reduced compared with that of the original communication graph which contains all links supported by the maximum transmission power. Among these 2D structures, some are planar structures (such as LMST, RNG, GG, and Delaunay-based graphs), some are power spanners (such as GG, Yao graph, CBTC, and Delaunay-based graphs), and some are with bounded node degree (such as Yao graph).

Besides these localized geometrical structures, there are also other various techniques proposed by researchers for topology control in 2D sensor networks, such as how to construct a virtual backbone for routing [27-30] and how to minimize the total transmission power while maintaining connectivity or other properties $[31,32,36,37]$. Since this article only focuses on localized geometrical structures with bounded node degree, we refer readers to some nice surveys [13-15] on topology control for more details.

\subsection{Topology control in 3D networks}

Although geometric topology control protocols have been well studied in 2D networks, the design of 3D topology control is surprisingly more difficult than the design in 2D. Current 2D methods cannot be directly applied in 3D networks. Wang et al. [39,40] proved that there is no embedding method mapping a $3 \mathrm{D}$ network into a $2 \mathrm{D}$ plane so that the relative scale of all edge length is preserved and all 2D geometric topology control protocols can still be applied for power efficiency. Thus, any simple mapping method from 3D to 2D does not work. On the other hand, many properties of 3D networks require additional computational complexity. Until recently, little research has been done on topology control for 3D wireless networks.

To solely achieve the connectivity of a 3D network, local minimum spanning tree (LMST) [21,22] may be the best choice, since it is very sparse (with a bounded node degree) and can be easily constructed even for 3D networks and preserve connectivity. However, LMST may have very large power stretch factor. Similarly, relative neighborhood graph (RNG) and Gabriel graph (GG) can be easily extended to $3 \mathrm{D}$, as shown in $[39,40]$. However, both of them do not have bounded node degree.

Bahramgiri et al. [38] generalized CBTC algorithm from $2 \mathrm{D}$ to $3 \mathrm{D}$ to preserve connectivity. Basically, each node $u$ increases its transmission power until there is no empty 3D-cone with angle degree $\alpha$, i.e., there exists at least a node in each 3D-cone of degree $\alpha$ centered at $u$, if $\alpha \leq \frac{2 \pi}{3}$. This algorithm can be extended to ensure $k$-connectivity with $\alpha \leq \frac{2 \pi}{3 k}$. Even though this approach can guarantee connectivity, the gap detection algorithm applied to check the existence of the empty 3D-cone of degree $\alpha$ is very complicated. The time complexity of the gap detection algorithm at a node $u$ is $O\left(d^{3} \log d\right)$, where $d$ is the node degree of $u$. Moreover, their method cannot bound node degree, as shown by [54].

Wang et al. $[39,40]$ proposed a set of 3D Yao-based topologies (FiYG and FlYG), which can be constructed locally and efficiently. They proved some nice properties of these 3D Yao-based structures, e.g., bounded node out-degree and constant power stretch factor. Later, Wang et al. [41] also extended all of their 3D structures to support fault tolerance.

Ghosh et al. [42] also presented two CBTC-based approaches for 3D wireless networks. Though the first approach, a heuristic based on 2D orthographic projections, can provide excellent performance in practice, it cannot guarantee connectivity for sure. In the second approach, a spherical Delaunay triangulation (SDT) is built to determine the existence of empty 3D cones. Although the second approach can guarantee connectivity of the network, the expense to construct the SDT is very high. Similarly, Poduri et al. [43] also used the spherical Delaunay triangulation to find the largest empty 3D cone in order to apply a CBTC-based topology control. The expense of SDT construction makes it inefficient in practice.

Recently, Kim et al. [44] proposed another localized Yao-based structure with Platonic solid (PYG). The basic idea is the same as the 3D Yao structures in $[39,40]$ except for the partition method of 3D cones. To construct PYG, each node divides the 3D sphere neighborhood into $k$ equal cones by using regular $k$-polyhedron and selects the neighbor that requires the lowest transmit power in each cone. The authors also consider the interference effects to adaptively choose the minimum transmit power and adjust the topology. However, such modification will break the connectivity and power spanner guarantees of 3D Yao structures.

Overall, all of these existing 3D structures cannot achieve bounded node degree. Notice that even though 3D Yao structures (including FiYG, FlYG, and PYG) can bound the node out-degree, their node in-degree could be as large as $O(n)$ where $n$ is the number of nodes in 
the networks. Faced up with this challenge, in this article we study how to efficiently construct 3D topology with bounded node degree to maintain connectivity, conserve energy and enable energy efficient routing.

\section{Degree-bounded 3D topologies: 3D symmetric Yao graph and 3D Yao \& reverse Yao graph}

In this section, we propose two general frameworks to build degree-bounded 3D topologies for wireless sensor networks. The proposed frameworks basically apply the existing 3D Yao structures via two techniques developed in $[19,23,55]$ for $2 \mathrm{D}$ topology control protocols. In addition, we provide detailed analysis on the degree bound and power stretch factor of the proposed new 3D topologies.

\subsection{Review of basic 3D Yao structures}

Our general frameworks to building degree-bounded 3D topologies are based on any existing 3D Yao structure (such as FiYG [39,40], FlYG [39,40] and PYG [44]). These 3D Yao structures use certain types of 3D cones to partition the transmission range of a node (which is a sphere), and inside each 3D cone the node only keeps a link to the nearest neighbor. Since the number of such 3D cones is bounded by a constant $k$, all of them can bound the node out-degree by $k$. Here, $k$ is a constant depending on which method and parameter are used. Basically, these structures can be categorized into two sets: fixed partition and flexible partition.

\section{D Yao structures based on fixed partition}

In fixed partition, 3D cones from one node do not intersect with each other and the partition method is the same for all nodes. In $[39,40]$, Wang et al. first proposed two methods to divides the transmission range of a node into certain number of 3D cones. Figure 1a,b illustrates these two methods, which divide the transmission ball into 32 and 56 cones, respectively. For each cone, node $u$ will choose the shortest edge $u v \in U B G$ among all edges emanated from $u$, if there is any, and add a directed link $\overrightarrow{u v}$. Ties are broken arbitrarily or by ID. The resulting directed graph are denoted by $F i Y G_{32}$ and $F i Y G_{56}$, respectively. Notice that these cones in FiY $G_{32}$ and $F i Y G_{56}$ are different and do not intersect with each other. In [44], Kim et al. then proposed another type of fixed partition method which divides the unit ball into $k$ equal cones by using a regular $k$-polyhedron and selects the nearest neighbor in each cone. The resulting directed graph is denoted by $P Y G_{k}$. Possible polyhedrons include tetrahedron, cube, octahedron, dodecahedron and icosahedron for $k=4,6,8,12,20$ respectively. Figure $1 \mathrm{c}, \mathrm{d}$ illustrates partition examples with a octahedron $k=8$ and a dodecahedron $k=12$. Notice that the cones in this method are with same shape/size and do not intersect with each other. All above methods based on fixed partition can be performed locally using 1-hop neighbor information and with $O(d)$ time, where $d$ is the number of 1-hop neighbors.

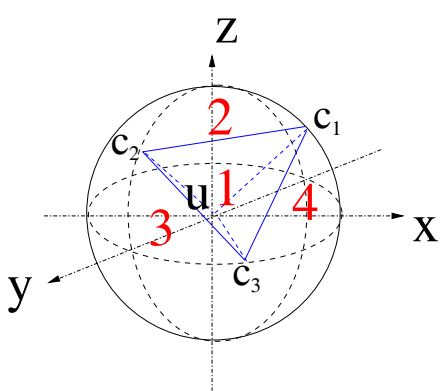

(a) $\mathrm{FiYG}_{32}$

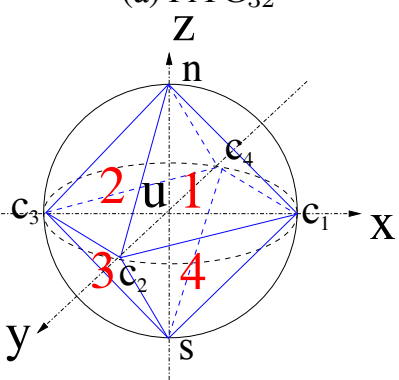

(b) $\mathrm{PYG}_{8}$

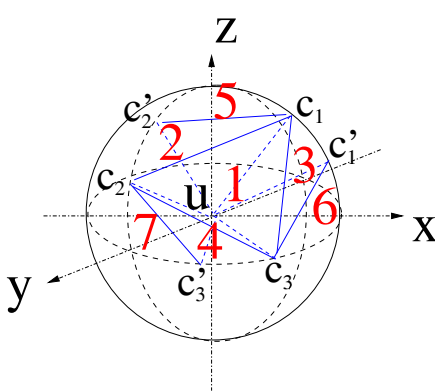

(b) FiYG $_{56}$

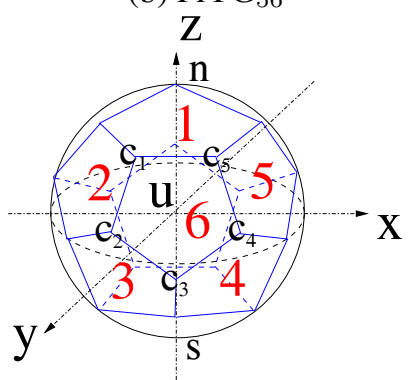

(c) $\mathrm{PYG}_{12}$

Figure 1 Definitions of 3D Yao Structures with fixed partitions. (a) and (b) show partitions of 1/8 of the ball in FiYG; (c) and (d) show partitions using a octahedron or a dodecahedron for PYG. 
3D Yao structures based on flexible partition

In flexible partition, identical 3D cones with a top angle $2 \theta$ are used to partition the transmission ball and where to define these cones depends on the locations of neighbors around node $u$. In $[39,40]$, Wang et al. proposed three different methods to perform the partition. However, they showed that the first method does not bound the node out-degree. Here, we just review their third method. Initially, all neighbors $v_{i}$ of node $u$ are unprocessed and ordered by the distance to $u$. The algorithm processes link $u v$ from the shortest link and follows an ascending order. When it processes $u v_{i}$, it defines the $3 \mathrm{D}$ cone $C_{u v_{i}}$ which uses $u v_{i}$ as its axis (as shown in Figure 2a), adds the link $u v_{i}$, and marks all other links in $C_{u v_{i}}$ as processed. We denote the final structure as $F l Y$ $G_{\theta}$ or FlYG when value of $\theta$ is clear. Algorithm 1 illustrates the detailed algorithm. The time complexity of this algorithm is $O(d \log d)$ due to the sorting. Notice that the $3 \mathrm{D}$ cones in this method are in the same size/ shape and can intersect with each other (as in Figure 2b). By using a volume argument ${ }^{\mathrm{a}}$, Wang et al. showed that the node out-degree of $F l Y G$ is bounded by

$k=\left\lceil\frac{2}{1-\cos \left(\frac{\theta}{2}\right)}\right]$. If $\theta=\pi / 4$, the degree bound is $\frac{2}{1-\cos \left(\frac{\theta}{2}\right)} \approx 26$, and if $\theta=\pi / 6$, the degree bound is 58 .

Algorithm 1. Construct 3D Yao Structure FlY G for Node $u$

Input: all neighbors $N_{U B G}(u)$ of node $u$ in UBG.

Output: neighbors $N_{F l Y G}(u)$ of $u$ in the constructed FlYG.

1: Sort all neighbors $v_{i} \in N_{U B G}(u)$ by its length such that $\left\|u v_{i}\right\| \leq\left\|u v_{i+1}\right\|$, where $i=1$ to $\left|N_{U B G}(u)\right|$.

2: Set PROCESSED $\left(v_{i}\right)=0$ for all neighbor $v_{i} \in N_{U B G}$ (u).

3: for $i=1$ to $\left|N_{U B G}(u)\right|$ do

4: if $\operatorname{PROCESSED}\left(v_{i}\right)=0$ then
5: As shown in Figure 2a, let $C_{u v_{i}}$ be the cone using $u v_{i}$ as the axis and $2 \theta$ as the top angle.

6: Keep $v_{i}$ as a neighbor of $u$ in FlYG, i.e., add $v_{i}$ in $N_{\text {FlYG }}(u)$.

7: Set $\operatorname{PROCESSED}(w)=1$ for every other neighbor $w$ inside $C_{u v i}$.

8: end if

9: end for

10: return $N_{F l Y G}(u)$

4.2 General frameworks to build 3D symmetric Yao graph and 3D Yao \& reverse Yao graph

Bounded out-degree from 3D Yao structures gives us advantages when apply several routing algorithms on these structures. However, possible unbounded indegree at some nodes will often cause large overhead or contention at those nodes which may make them exhausted earlier than other nodes. Therefore it is often imperative to construct a sparse network topology such that both the in-degree and the out-degree are bounded by a constant while it is still power spanner. Hereafter, we define a general function 3D-YAO-Structure() which can generate the neighbor set of 3D Yao structure at node $u$ given the current neighbor set of $u$. The 3DYAO-Structure() function can be any generation methods of existing Yao-based 3D structures. We use YG to denote the generated 3D Yao structure.

The first set of 3D topologies is 3D symmetric Yao graph (SYG), an undirected graph, which guarantees that the node degree is at most $k$. It first applies the 3D Yao structure to select the closest node in each 3D cone. An link $u v$ is selected to graph SYG if and only if both $u$ and $v$ are selected to be kept by each other in YG, i.e., $v \in N_{Y G}(u)$ and $u \in N_{Y G}(v)$. See Figure 3a,b for illustrations. Algorithm 2 shows the framework. It is clear that only one-hop information is used and total $O$ (n) of messages are used. Thus, the SYG can be built locally and efficiently. Notice that similar idea has been used in 2D networks $[23,55,56]$.

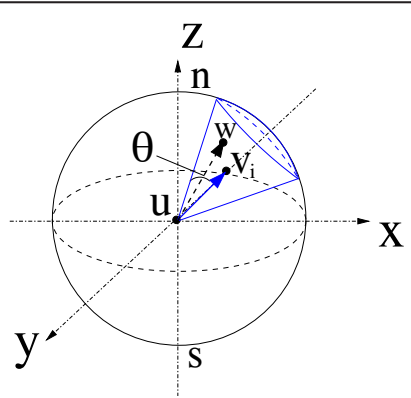

(a) $3 \mathrm{D}$ cone defined by $u v_{i}$

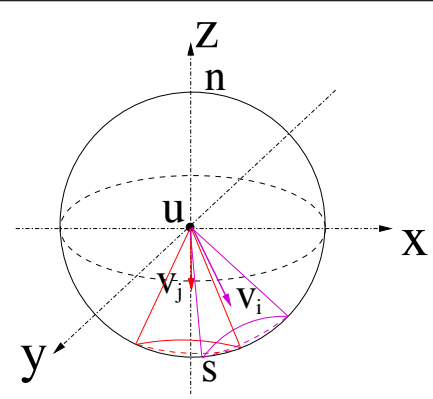

(b) Possible overlapping of cones

Figure 2 Definitions of 3D Yao structures with flexible partitions. 


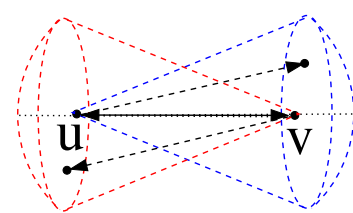

(a) $u v \in \mathrm{SYG}$

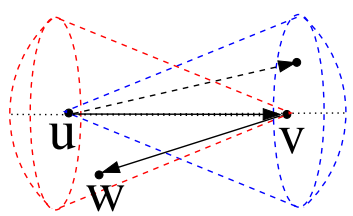

(b) $u v \notin \mathrm{SYG}$

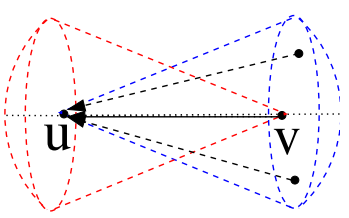

(c) $\overleftarrow{u v} \in \mathrm{YYG}$

Figure 3 Illustrations of 3D Yao Structures with bounded degree. (a) and (b) show 3D symmetric Yao graph; (c) shows 3D Yao and reverse Yao graph.

Algorithm 2. Building 3D symmetric Yao graph at node $u$

Input: all neighbors $N_{U B G}(u)$ of node $u$ in UBG.

Output: neighbors $N_{S Y} G(u)$ of $u$ in the constructed SYG.

1: $N_{Y G}(u)=3 \mathrm{D}-\mathrm{YAO}-\operatorname{Structure}\left(N_{U B G}(u)\right)$.

2: Broadcast $N_{Y G}(u)$ to all neighbors $N_{U B G}(u)$.

3: for all node $v \in N_{Y G}(u)$ do

4: if $u \in N_{Y G}(v)$ then

5: Keep $v$ as a neighbor of $u$ in SYG, i.e., add $v$ in $N_{S Y}$ ${ }_{G}(u)$.

6: end if

7: end for

8: return $N_{S Y G}(u)$

Algorithm 3. Building 3D Yao and reverse Yao graph at node $u$

Input: all neighbors $N_{U B G}(u)$ of node $u$ in UBG.

Output: neighbors $N_{Y Y G}(u)$ of $u$ in the constructed YYG.

1: $N_{Y G}(u)=3 \mathrm{D}-\mathrm{YAO}-$ Structure $\left(N_{U B G}(u)\right)$.

2: Broadcast $N_{Y G}(u)$ to all neighbors $N_{U B G}(u)$.

3: Let $N_{Y G}^{i n}(u)$ be the set of $u$ 's incoming neighbors, i. e., all node $v$ satisfying $u \in N_{Y G}(v)$.

4: $N_{Y Y G}^{i n}(u)=3 \mathrm{D}$ - YAO - Structure $\left(N_{Y G}^{\text {in }}(u)\right)$.

5: Broadcast $N_{Y Y G}^{i n}(u)$ to all neighbors $N_{U B G}(u)$.

6: for all node $v \in N_{Y G}(u)$ do

7: if $u \in N_{Y Y G}^{i n}(v)$ then

8: Keep $v$ as a neighbor of $u$ in YYG, i.e., add $v$ in $N_{Y}$ ${ }_{Y G}(u)$.

9: end if

10: end for

11: return $N_{Y Y G}(u)$

The second set of 3D topologies is 3D Yao and reverse Yao graph (YYG), a directed graph, which guarantees that both node in-degree and node out-degree are at most $k$. The basic idea is to apply reverse 3D Yao structure on YG to bound the node in-degree. Node $u$ chooses a node $v$ from each 3D cone, if there is any, so the incoming link $\overleftarrow{u v}$ in YG has the smallest length among all incoming links from YG in that cone as shown in Figure 3c. Similar idea has been used for 2D networks by $[16,19,57]$. Algorithm 3 shows the detailed algorithm. 3D YYG can be built locally and efficiently with only 1-hop neighbor information and linear number of messages.

\subsection{Performance analysis of 3D SYG and 3D YYG}

We are now ready for providing some analysis on the 3D structures built by our general frameworks. We will use two basic properties of the underlying 3D Yao structures: (1) the out-degree of 3D YG is bounded by $k$; and (2) if a link $u v \in U B G$ is not kept in 3D YG, there must exist a shorter link $u w$ kept in 3D YG and $\angle v u w<\theta$. Here $\theta$ is the largest angle possible in a 3D cone in FiYG or the half of the top angle of the 3D cone in FlYG. For simplification, we assume the maximum transmission range $R=1$.

Theorem 1 Both $3 D$ SYG and $3 D$ YYG are strongly connected if the original $3 D$ UBG is connected and the angle parameter $\theta$ in $3 D Y G$ is bounded by $\pi / 3$.

PROOF. We first prove the connectivity of 3D SYG, which is equivalent to prove that there is a directed path from $u$ to $v$ in SYG for any two nodes $u$ and $v$ with $\|u v\| \leq 1$. We prove this claim by an induction over the distance $\|u v\|$ between nodes $u$ and $v$. First, note that the edge between the closest pair of nodes must be kept in SYG. Assume that the claim is true for all links less than $\|u v\|$. Now we consider nodes $u$ and $v$. If $u v$ is kept in SYG, the claim is true. If $u v$ is not in SYG, there must a node $w$ inside one of 3D cones at $u$ or $v$ who causes the deletion of $u v$. Assume $w$ and $v$ are in the same cone of $u$ and $\|u w\|<\|u v\|$. Because the angle $\angle w u v$ is less than $\theta \leq \frac{\pi}{3}$, we have $\|v w\|<\|u v\|$. By induction there is a path from $u$ to $w$ and a path from $w$ to $v$ in SYG. Therefore a path from $u$ to $v$ exists in SYG. This finishes the proof for SYG.

We next prove that SYG is a subgraph of YYG, which then can imply the connectivity of 3D YYG automatically. Assume that there exists a link $u v$ in SYG but not in YYG. From the definition of SYG, we know link $u v$ is selected by both $u$ and $v$ in 3D YG. Then if we apply reverse Yao structure on incoming neighbor of 3D YG (as Line 4 in Algorithm 3), $u v$ will also be selected by node $v$. Thus, $u v$ must be in YYG. This is a 
contradiction. Therefore, YYG is a supergraph of SYG and fully connected. $\square$

The above theorem shows that our proposed algorithms can guarantee the connectivity of the final structure given that the underlying UBG is connected. In other words, our algorithms only remove links whose deletions will not affect the connectivity. Therefore, if the underlying UBG is already very sparse, our algorithms will not remove many links or even any links. But if the UBG is denser, more links will be removed by our proposed algorithms. This has been confirmed by our simulations (presented in Section 5). Notice that how to guarantee the connectivity of the UBG (where every node uses the maximum transmission power) is beyond the scope of this article. However, there are several existing studies $[31,33-35,54]$ on how to set up the maximum transmission power to guarantee the network connectivity.

Theorem 2 The node degree of $3 D$ SYG is bounded by $k$ while both node out-degree and in-degree of $3 D Y Y G$ is bounded by $k$, where $k$ is the degree bound of underlying $3 D Y G$.

PROOF. This theorem is straightforward from the construction methods of SYG and YYG. Both methods first apply 3D YG. Since each node has at most $k$ 3D cones during this construction, the out-degree is bounded by $k$. For 3D SYG, a link is kept only if both endpoints keep it in 3D YG. Thus, the node degree of SYG is obviously bounded by $k$. For 3D YYG, the second round of 3D YG is applied to incoming links, thus the node in-degree is also bounded by $k$. Notice that in 3D YYG, the out-degree and in-degree neighbors of a node may be different set of nodes. $\square$

Theorem 3 The $3 D$ SYG is not a power spanner of $U B G$, while $3 D Y Y G$ is a power spanner of $U B G$ when $\beta$ $\geq 3$ and $\theta<\pi / 3$.

PROOF. The first half of this theorem can be directly obtained from a result by Grunewald et al. [16]. They basically show how to construct a counter example of a 2D network in which SYG is not a power spanner. Since the $2 \mathrm{D}$ network is a special case of $3 \mathrm{D}$ networks, the same counter example works for 3D networks.
The proof of power spanner property of YYG is much challenging, even in 2D. Jia et al. [58] first proved that 2D YYG is a power spanner when $\theta \leq \pi / 60$ (i.e., $k \geq$ $120)$. It seems that their proof might be extended to $3 D$ case, however, the node degree bound will be huge (larger than $\left.\frac{4 \pi / 3}{2 \pi(1-\cos (\theta / 2)) / 3} \geq 5836\right)$. Thus it is not very useful in practice. Schindelhauer et al. [59] then proved that $2 \mathrm{D}$ YYG is a power spanner with power spanning ratio $(8 c+1)^{2} \frac{(2 c)^{\beta}}{1-2^{(2-\beta)}}$ for $\beta>2$ when $k>6$.

Here $c=\frac{1}{1-2 \sin (\pi / k)}$. They proved this by first proving that 2D YYG is a weak $c$-spanner. In a weak $c$-spanner, between any two nodes there exists a path which remains within a disk or sphere of radius $c$-times the Euclidean distance between them. Their proof of weak spanner property of YYG can also be extended to 3D YYG with $\theta<\pi / 3$. However, to further extend it to 3D power spanner, it requires $\beta \geq 3$. More specifically, 3D YYG is a power spanner with power spanning ratio $(8 c+1)^{3} \frac{\left(2 c^{\beta}\right.}{1-2^{(3-\beta)}}$ for $\beta>3$ or $O\left(c^{12}\right)$ for $\beta=3$ when $\theta<$ $\pi / 3$. Therefore, we can claim that $3 \mathrm{D}$ YYG is a power spanner for $\beta \geq 3$ and $\theta<\pi / 3$. When $2 \leq \beta<3$, the power spanner property is still open. $\square$

Table 1 summarizes the properties of all proposed and existing 3D topologies. Notice that the time complexity of 3D Yao-based structures is $O(d)$ for fixed partitions and $O(d \log d)$ for flexible partitions. Here $d$ is the number of 1-hop neighbors. In addition, the power stretch factor of 3D YYG is $O(1)$ only for $\beta \geq 3$, but still open for $2 \leq \beta<3$.

\section{Simulation results}

In order to evaluate the performance of our new 3D topologies with bounded degree, we conduct simulations by generating random 3D sensor networks. In our experiments, we randomly generate a set $V$ of $n$ wireless nodes and the UBG, then test the connectivity of UBG. If it is connected, we construct different localized 3D topologies proposed in this article and some existing ones, and measure the node degree as well as power efficiency of these topologies. We repeat the experiment

Table 1 Property summary for existing and proposed 3D topologies

\begin{tabular}{ccccccc}
\hline 3D structure & connectivity & out-degree & in-degree & $\begin{array}{c}\text { power } \\
\text { stretch } \\
\text { factor }\end{array}$ & message & time \\
\hline 3D LMST & yes & $O(1)$ & $O(1)$ & $O(n)$ & $O(n)$ & $O\left(d^{2}\right)$ \\
3D RNG & yes & $O(n)$ & $O(n)$ & $O(n)$ & $O(n)$ & $O(d)$ \\
3D GG & yes & $O(n)$ & $O(n)$ & 1 & $O(n)$ & $O(d)$ \\
3D YG & yes & $O(1)$ & $O(n)$ & $O(1)$ & $O(n)$ & $O(d)$ or $O(d \log d)$ \\
3D SYG & yes & $O(1)$ & $O(1)$ & $O(n)$ & $O(n)$ & $O(d)$ or $O(d \log d)$ \\
3D YYG & yes & $O(1)$ & $O(1)$ & $O(1)$ & $O(n)$ & $O(d)$ or $O(d \log d)$ \\
\hline
\end{tabular}


for multiple times and report the average or maximum values of these metrics in all of the simulations.

We evaluate the following localized 3D topologies:

- Sparse topologies: RNG and GG;

- 3D Yao structures: FiYG $32, \mathrm{PYG}_{8}, \mathrm{PYG}_{12}, \mathrm{FlYG} \pi / 4$, and FlYG $\pi / 6$;

- 3D symmetric Yao structures: FiSYG $32, \mathrm{PSYG}_{8}$, $\mathrm{PSYG}_{12}, \mathrm{FlSYG}_{\pi / 4}$, and FlSYG $\pi / 6$;
- 3D Yao \& reverse Yao Structures: FiYYG $32, \mathrm{PYYG}_{8}$, $\mathrm{PYYG}_{12}, \mathrm{FlYTG}_{\pi / 4}$, and FlYYG $\pi / 6$;

Here, "SYG and "YYG are the symmetric Yao structure and Yao \& Reverse Yao structure based on the corresponding underlying 3D Yao structures, respectively.

Figure 4 shows a set of topologies generated for a UBG with 100 wireless nodes. In the experimental results presented here, we generate $n$ random wireless

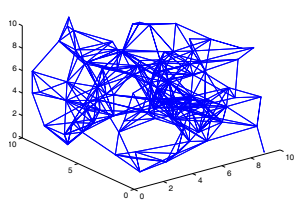

(a) UBG

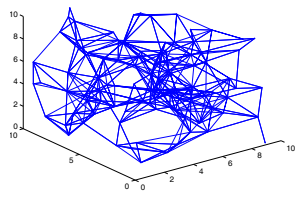

(d) FiYG $_{32}$

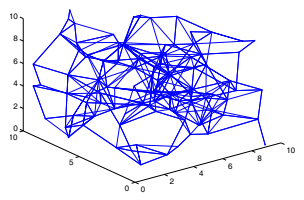

(g) $\mathrm{FlYG}_{\pi / 4}$

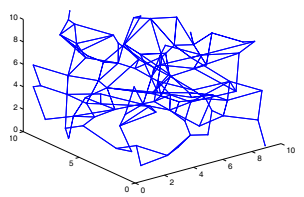

(j) $\mathrm{PSYG}_{8}$

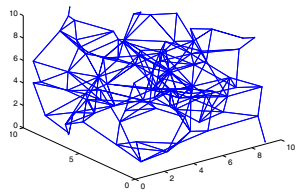

(m) $\mathrm{FlSYG}_{\pi / 6}$

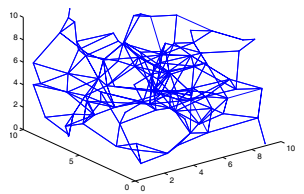

(p) $\mathrm{PYYG}_{12}$

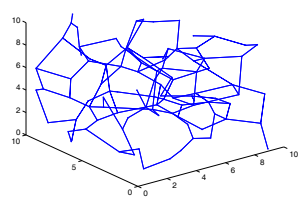

(b) RNG

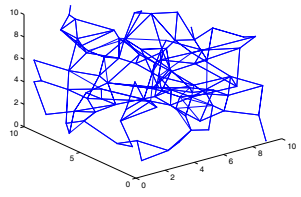

(e) $\mathrm{PYG}_{8}$

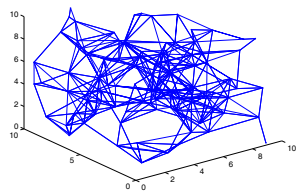

(h) $\mathrm{FlYG}_{\pi / 6}$

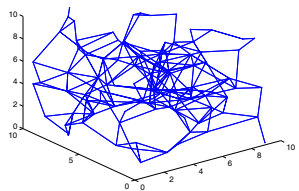

(k) $\mathrm{PSYG}_{12}$

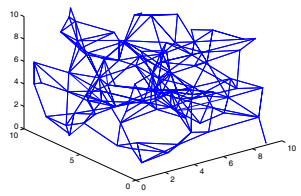

(n) FiYYG 32

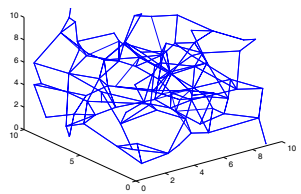

(q) $\mathrm{FlYYG}_{\pi / 4}$

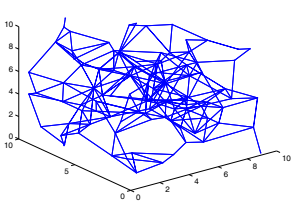

(c) GG

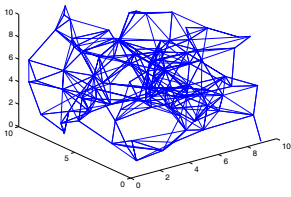

(f) $\mathrm{PYG}_{12}$

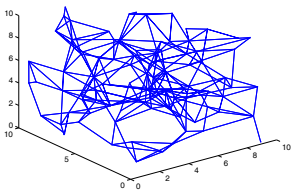

(i) $\mathrm{FiSYG}_{32}$

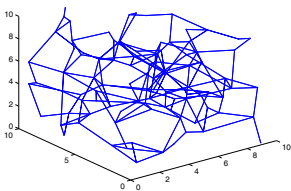

(1) $\mathrm{FlSYG}_{\pi / 4}$

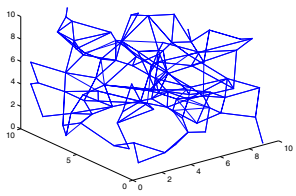

(o) $\mathrm{PYYG}_{8}$

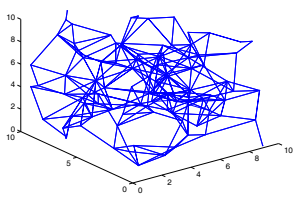

(r) FlYYG $_{\pi / 6}$

Figure 4 3D topologies generated from the same UBG 
nodes in a $10 \times 10 \times 10$ cube; the maximum transmission range $R$ is set to $\sqrt{10}$ and the power constant $\beta=$ 2 , thus the maximum transmission power $P_{\max }=R^{2}=$ 10. It is clear that RNG is the sparsest structure, however, we know that it is not a power spanner of UBG. For all Yao structures, the more 3D cones defined (larger $k$ or smaller $\theta$ ) the denser the topology is. For the structures based on the same 3D Yao construction
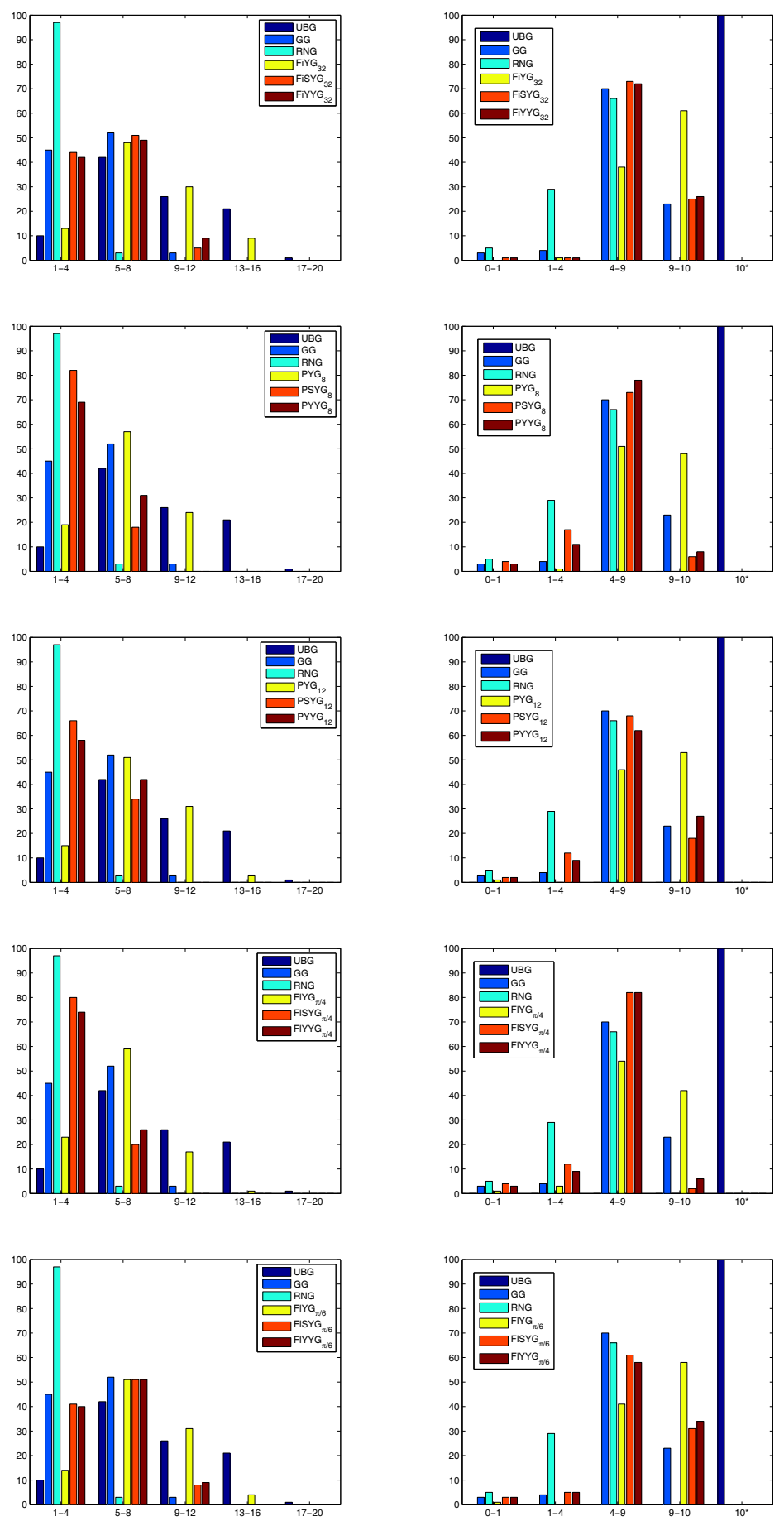

(a) node degree

(b) transmission power

Figure 5 Degree distributions and transmission power distributions. (a) shows the distributions of node degrees of the UBG and other topologies; (b)shows the distributions of final assigned transmission power levels of nodes. 
method, symmetric Yao structure is sparser than Yao \& reverse Yao structure.

For the same instance, we plotted the node degrees of different topologies in Figure 5a. It is clear that UBG has more nodes with high node degree. While RNG, GG and all Yao structures can drastically reduce node degrees. Figure $5 \mathrm{~b}$ shows the assigned minimal power levels for all the nodes. Here we assume that each node

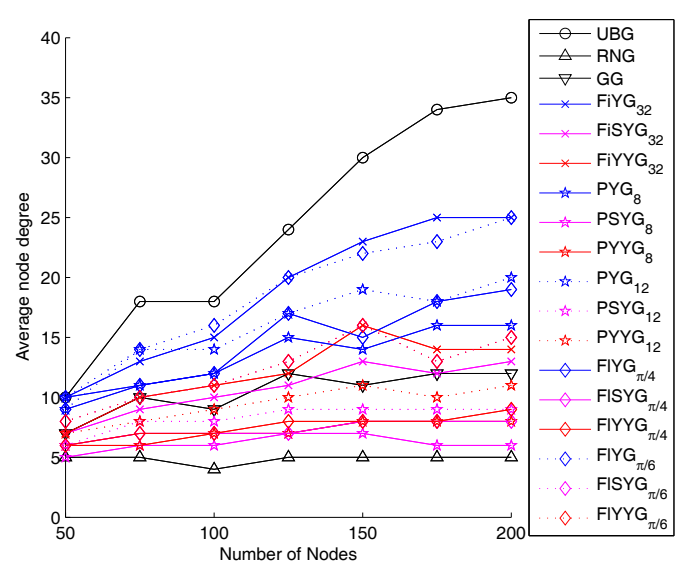

(a) avg-node-degree

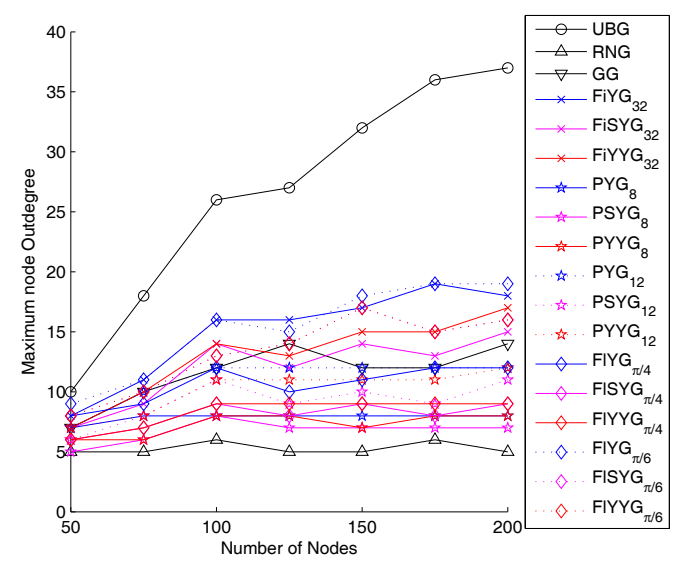

(c) max-node-out-degree

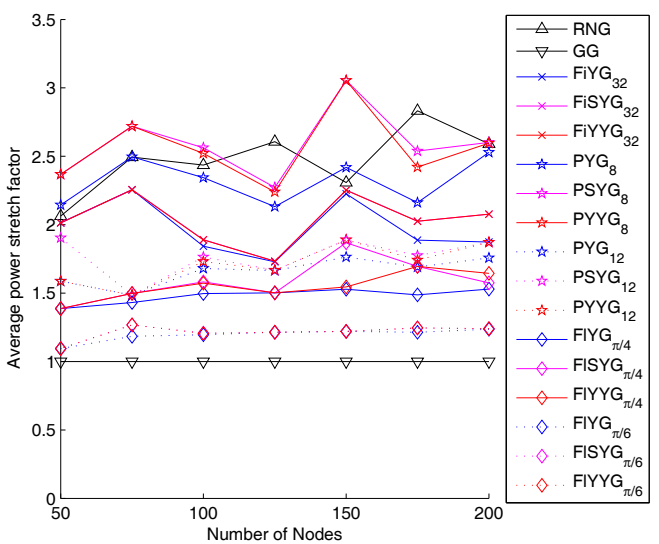

(e) avg-power-stretch-factor

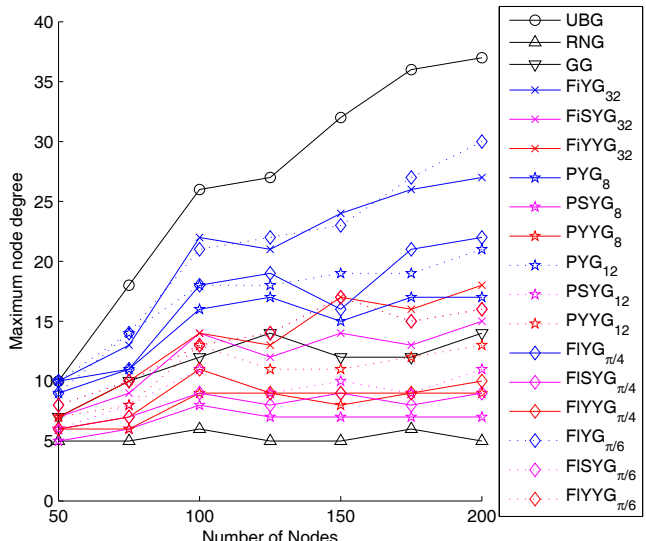

(b) max-node-degree

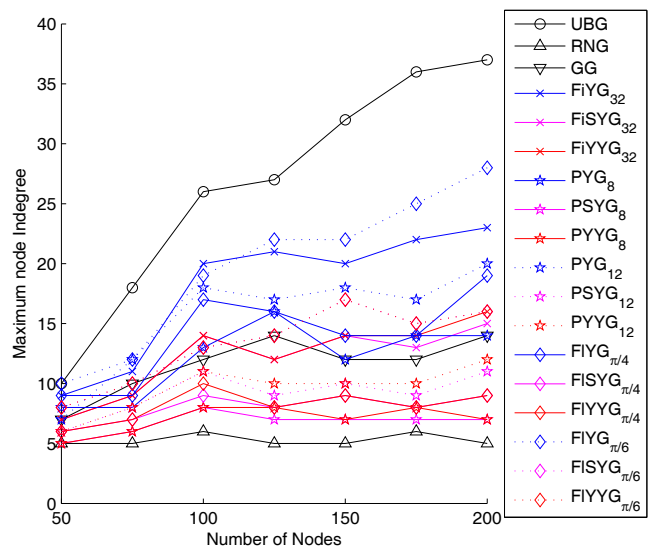

(d) max-node-in-degree

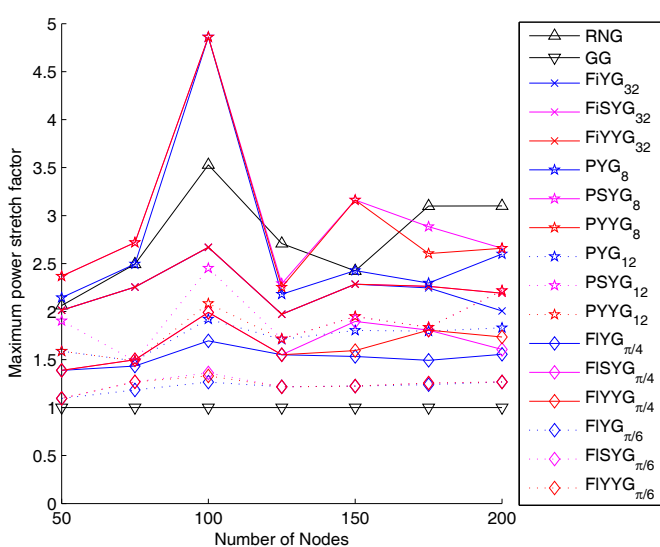

(f) max-power-stretch-factor

Figure 6 Results when the number of sensor nodes increasing from 50 to 200 
can shrink its power level to support the longest link in the generated topology. Clearly, no node needs to transmit at its maximum power level anymore in these sparse topologies. RNG and GG use the smallest power level, since they are the sparest graphs. All Yao structures can also save a lot of energy compared with UBG. Clearly, more 3D cones defined in Yao structure, less energy it saves and larger node degree it has.

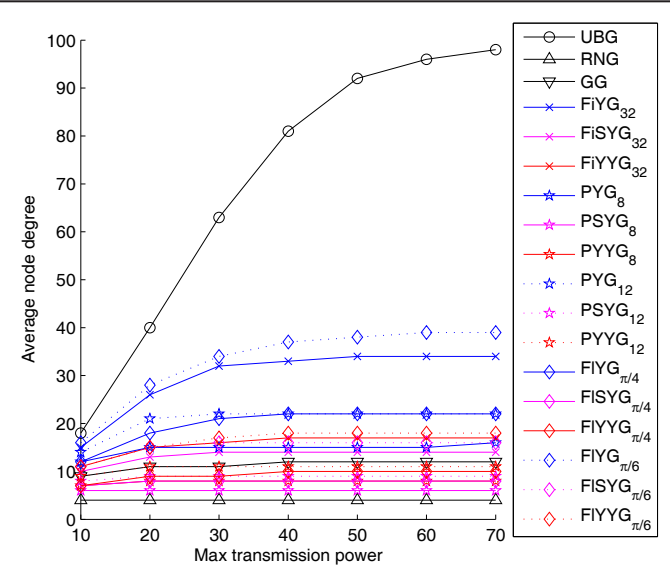

(a) avg-node-degree

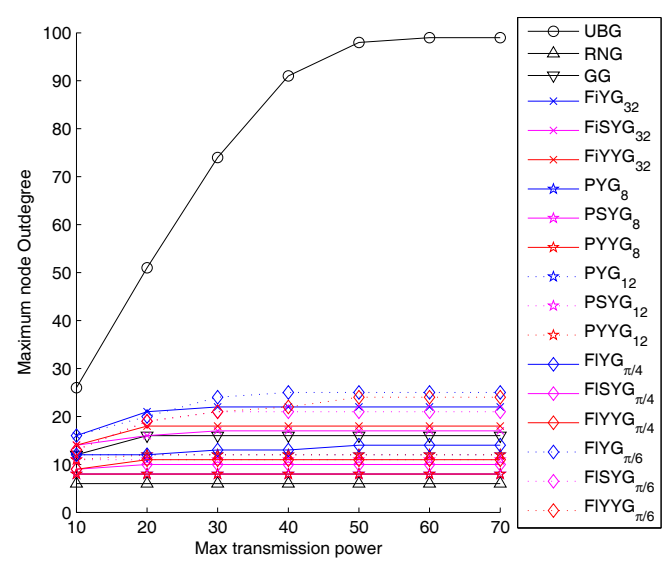

(c) max-node-out-degree

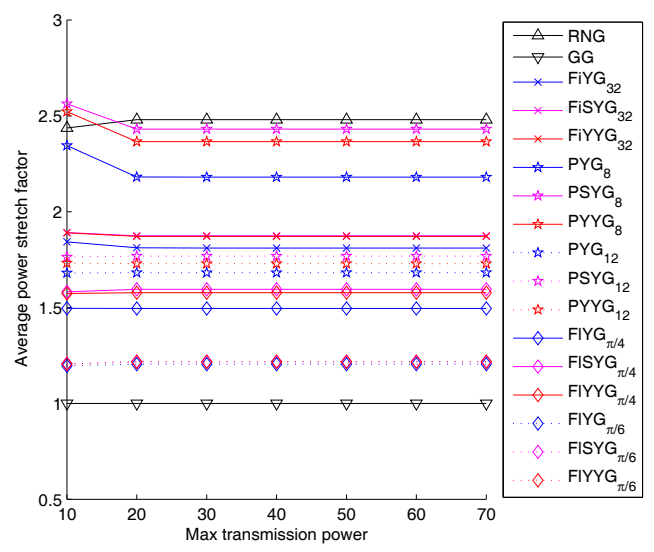

(e) avg-power-stretch-factor

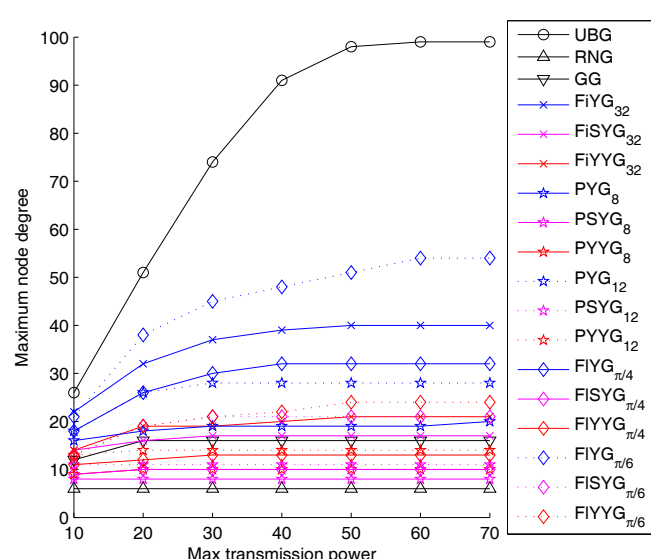

(b) max-node-degree

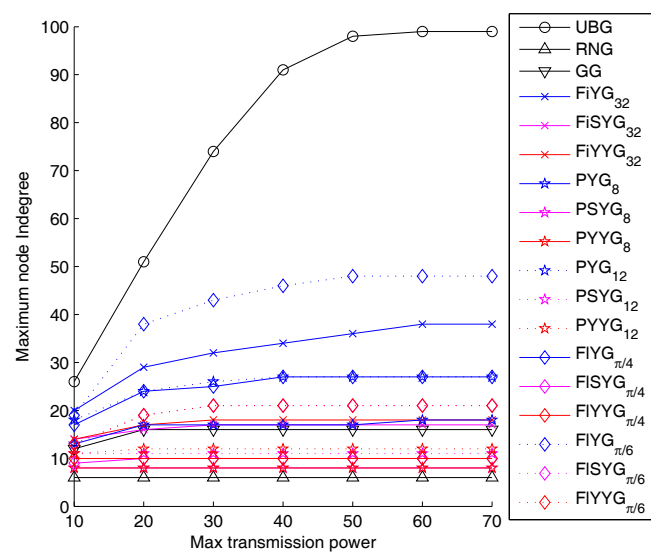

(d) max-node-in-degree

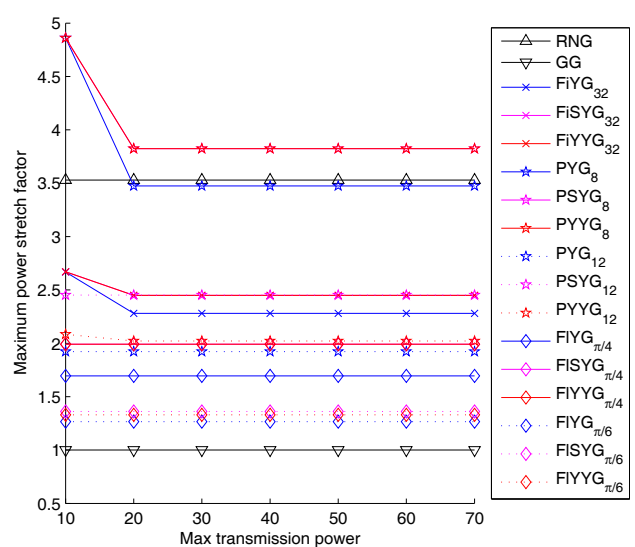

(f) max-power-stretch-factor

Figure 7 Results when the maximum transmission power increasing from 10 to 70 
We vary the number of nodes $n$ in the network from 50 to 200 , where 100 vertex sets are generated for each case. The average and the maximum are computed over all these 100 vertex sets. All experimental results are plotted in Figure 6.

The node degree of wireless networks should not be too large to avoid interference, collision, and overhead. It should not be too small either: a low node degree usually implies that the network has a low fault tolerance and tends to increase the overall network power consumption as longer paths may have to be taken. Figure 6a shows all localized 3D topologies have lower average degrees compared with UBG and keep small degrees when the degree of UBG becomes denser and denser as the number of nodes in the network increases. Clearly, Yao structures with larger number of 3D cones lead to denser structures, while RNG is much sparser than most of Yao-based structures. Notice that the structures sparser than GG are Symmetric Yao structures and Yao \& Reverse Yao structures of $\mathrm{PYG}_{12}, \mathrm{PYG}_{8}$ and $\mathrm{FlYG}_{\pi / 4}$ since these structures have smallest $3 \mathrm{D}$ cones. These can also be verified by Figure 4 and $6 \mathrm{~b}$ (the maximum node degree). Figure $6 \mathrm{c}$ shows that the maximum node out-degree of these localized 3D-topologies are small. The out-degrees of all Yao structures are smaller than the theoretical bounds. Figure $6 \mathrm{~d}$ also gives the maximum node in-degree of these topologies which are a little bit larger than their out-degrees. It is clear that both symmetric Yao structures and Yao \& reverse Yao structures have smaller in-degrees than 3D Yao structures. Remember that theoretically they can bound the node in-degree. The results also showed that RNG and GG do not have large degrees and 3D Yao structures do not have large in-degree in this experiment, and the reasons are that the nodes are distributed randomly in the area. In real life, the network may not be distributed randomly, so it is possible that RNG and GG have large degrees and 3D Yao structures have large indegrees. Such examples and simulation results can be found in [19].

Besides connectivity, the most important design metric of wireless networks is perhaps power efficiency, because it directly affects both nodes and network lifetime. Figure $6 \mathrm{e}$,f show all proposed structures have small power stretch factors even when the network is very dense. Notice that Yao structures based on $\mathrm{PYG}_{8}$ and RNG have a little bit higher stretch factor than GG and other Yao-based structures, however, their maximum power stretch factors are still smaller than 5 . The reason again is that the nodes are distributed randomly in the area and the number of nodes is not too large. As we expected, GG has a power stretch factor of one and all power stretch factors of 3D Yao structures are smaller than their theoretical bounds if they have ones.
Among all 3D Yao structures, $\mathrm{FlYG}_{\pi / 6}$ and $\mathrm{FlYYG}_{\pi / 6}$ have the smallest stretch factor, since they are the densest 3D Yao structures.

We also conduct simulations on random networks with fixed 100 nodes and various maximum transmission power $P_{\max }$ is from 10 to 70 . Simulation results are plotted in Figure 7. Basic conclusions from this set of simulations are coherent with previous simulation set.

\section{Conclusion}

Topology control for 3D wireless sensor networks has been well studied recently and different 3D geometric topologies were proposed to achieve the sparseness and power efficiency. However, most of them cannot bound the node degree. Even though some of 3D structures based on Yao graph can bound the node out-degree, they may still lead to large in-degree at some nodes. Therefore, in this article, we propose two general frameworks to build degree-bounded 3D topologies for wireless sensor networks. These frameworks can use all existing Yao-based 3D structures as the underlying methods, and only use local information with linear number of messages. We show some of them can also guarantee the constant power stretch factor in 3D. Our simulation results confirm the good performance of our new 3D topologies.

\section{Endnote}

${ }^{\mathrm{a}}$ As shown in Figure 2b, the angle between two neighbors in $\mathrm{FlYG}_{\theta}$ must be larger than $\theta$, i.e., $\angle v_{i} u v_{j}<\theta$.

\section{Acknowledgements}

The study of F. Li was partially supported by the National Natural Science Foundation of China (NSFC) under Grant No. 60903151, Beijing Natural Science Foundation under Grant No. 4122070, and the Scientific Research Foundation for the Returned Overseas Chinese Scholars, State Education Ministry. The study of Y. Wang was supported in part by the US National Science Foundation (NSF) under Grant No. CNS-0721666, CNS-0915331, and CNS-1050398.

\section{Author details}

${ }^{1}$ School of Computer Science, Beijing Institute of Technology, Beijing, 100081, China ${ }^{2}$ Department of Computer Science, University of North Carolina at Charlotte, Charlotte, NC 28223, USA

\section{Competing interests}

The authors declare that they have no competing interests.

Received: 3 August 2011 Accepted: 1 May 2012 Published: 1 May 2012

\section{References}

1. CF Huang, YC Tseng, LC Lo, The coverage problem in three-dimensional wireless sensor networks, in Proc of IEEE Globecom 2004, vol. 5. Dallas, Texas, USA, pp. 3182-3186 (2004)

2. M Watfa, S Commuri, Optimal 3-dimensional sensor deployment strategy, in Proc of 3rd IEEE Consumer Communications and Networking Conference (CCNC 2006), vol. 2. Las Vegas, Nevada, USA, pp. 892-896 (2006)

3. M Watfa, S Commuri, The 3-dimensional wireless sensor network coverage problem, in Proceedings of the 2006 IEEE International Conference on 
Networking, Sensing and Control (ICNSC'06), Ft Lauderdale, Florida, USA, pp. 856-861 (2006)

4. SMN Alam, ZJ Haas, Coverage and connectivity in three-dimensional networks, in Proceedings of the 12th ACM Annual International Conference on Mobile Computing and Networking (MobiCom'06), New York, NY, USA, pp. 346-357 (2006)

5. V Ravelomanana, Extremal properties of three-dimensional sensor networks with applications. IEEE Trans Mobile Comput. 3(3), 246-257 (2004). doi:10.1109/TMC.2004.23

6. D Pompili, T Melodia, Three-dimensional routing in underwater acoustic sensor net-works, in Proceedings of the 2nd ACM international workshop on Performance evaluation of wireless ad hoc, sensor, and ubiquitous networks (PE-WASUN), Montreal, Canada, pp. 214-221 (2005)

7. P Xie, JH Cui, L Lao, VBF: vector-based forwarding protocol for underwater sensor networks, in Proceedings of the 5th international IFIP-TC6 conference on Networking Technologies (Networking'06), Coimbra, Portugal, pp. 1216-1221 (2006)

8. G Kao, T Fevens, J Opatrny, Position-based routing on 3-D geometric graphs in mobile ad hoc networks, in Proceedings of the 17th Canadian Conference on Computational Geometry (CCCG 2005), Ontario, Canada, pp. 88-91 (2005)

9. A Abdallah, T Fevens, J Opatrny, Power-aware 3D position-based routing algorithm for ad hoc networks, in Proc of 2007 IEEE International Conference on Communications (ICC), Glasgow, Scotland, pp. 3130-3135 (2007)

10. F Li, S Chen, Y Wang, J Chen, Load balancing routing in three dimensional wireless networks, in Proc of 2008 IEEE International Conference on Communications (ICC), Beijing, China, pp. 3073-3077 (2008)

11. C Liu, J Wu, Efficient geometric routing in three dimensional ad hoc networks, in Proc of 28th Annual IEEE Conference on Computer Communications (INFOCOM), Mini-conference, Rio, Brazil, pp. 2751-2755 (2009)

12. Y Wang, CW Yi, M Huang, F Li, Three dimensional greedy routing in largescale random wireless sensor networks. Ad Hoc Netw. (2011, to appear)

13. R Rajaraman, Topology control and routing in ad hoc networks: a survey. SIGACT News. 33, 60-73 (2002). doi:10.1145/564585.564602

14. P Santi, Topology Control in Wireless Ad Hoc and Sensor Networks, (JohnWiley \& Sons, Chichester, UK, 2005)

15. Y Wang, Topology control for wireless sensor networks, in Wireless Sensor Networks and Applications, ed. by Y Li, M Thai, W Wu (Springer, New York, USA, 2007)

16. M Grünewald, T Lukovszki, C Schindelhauer, K Volbert, Distributed maintenance of resource efficient wireless network topologies, in Proc of the 8th European Conference on Parallel Computing (Euro-Par'02), Paderborn, Germany, pp. 935-946 (2002)

17. L Li, JY Halpern, P Bahl, YM Wang, R Wattenhofer, Analysis of a cone-based distributed topology control algorithms for wireless multi-hop networks, in Proc of ACM Symposium on Principle of Distributed Computing (PODC), Newport, Rhode Island, USA, pp. 264-273 (2001)

18. XY Li, PJ Wan, Y Wang, Power efficient and sparse spanner for wireless ad hoc networks, in Proc of IEEE Int Conf on Computer Communications and Networks (ICCCNO1), Scottsdale, Arizona, USA, pp. 564-567 (2001)

19. XY Li, PJ Wan, Y Wang, O Frieder, Sparse power efficient topology for wireless networks, in Proc of IEEE Hawaii Int Conf on System Sciences (HICSS), Big Island, HI, USA, pp. 3839-3848 (2002)

20. R Wattenhofer, L Li, P Bahl, YM Wang, Distributed topology control for wireless multihop ad-hoc networks, in Proc of the 20th Annual Joint Conference of the IEEE Computer and Communications Societies (INFOCOM), vol. 3. Anchorage, Alaska, USA, pp. 1388-1397 (2001)

21. N Li, JC Hou, L Sha, Design and analysis of a MST-based topology control algorithm, in Proc of the 23th Annual Joint Conference of the IEEE Computer and Communications Societies (INFOCOM), vol. 3. San Francisco California, USA, pp. 1702-1712 (2003)

22. XY Li, Y Wang, WZ Song, Applications of k-Local MST for topology control and broadcasting in wireless ad hoc networks. IEEE Trans Parallel Distrib Syst. 15(12), 1057-1069 (2004). doi:10.1109/TPDS.2004.77

23. $X Y$ Li, I Stojmenovic, Y Wang, Partial delaunay triangulation and degree limited localized bluetooth multihop scatternet formation. IEEE Trans Parallel Distrib Syst. 15(4), 350-361 (2004). doi:10.1109/TPDS.2004.1271184

24. XY Li, G Calinescu, PJ Wan, Y Wang, Localized delaunay triangulation with application in wireless ad hoc networks. IEEE Trans Parallel Distrib Process. 14(10), 1035-1047 (2003). doi:10.1109/TPDS.2003.1239871
25. J Gao, L Guibas, J Hershburger, L Zhang, A Zhu, Geometric spanner for routing in mo-bile networks, in Proceedings of the 2nd ACM Symposium on Mobile Ad Hoc Networking and Computing (MobiHoc 01), Long Beach, CA, USA, pp. 45-55 (2001)

26. R Ramanathan, R Hain, Topology control of multihop wireless networks using transmit power adjustment, in Proc of the 19th Annual Joint Conference of the IEEE Computer and Communications Societies (INFOCOM), vol. 2. Tel Aviv, Israel, pp. 404-413 (2000)

27. N Nikaein, C Bonnet, Topology management for improving routing and network performances in mobile ad hoc networks. Mob Netw Appl. 9(6), 583-594 (2004)

28. L Bao, JJ Garcia-Luna-Aceves, Topology management in ad hoc networks, in Proceedings of the 4th ACM international symposium on Mobile Ad Hoc Networking \& Computing (MobiHoc), pp. 129-140 (2003)

29. B Liang, ZJ Haas, Virtual backbone generation and maintenance in ad hoc network mobility management, in Proc of the 19th Annual Joint Conference of the IEEE Computer and Communications Societies (INFOCOM), vol. 3. Tel Aviv, Israel, pp. 1293-1302 (2000)

30. K Alzoubi, XY Li, Y Wang, PJ Wan, O Frieder, Geometric spanners for wireless ad hoc networks. IEEE Trans Parallel Distrib Process. 14(4), 408-421 (2003). doi:10.1109/TPDS.2003.1195412

31. AE Clementi, G Huiban, P Penna, G Rossi, YC Verhoeven, Some recent theoretical advances and open questions on energy consumption in adhoc wireless networks, in Proc of 3rd Workshop on Approximation and Randomization Algorithms in Communication Networks (ARACNE), vol. 15. Rome, Italy, pp. 23-38 (2002)

32. L Lloyd, R Liu, MV Marathe, R Ramanathan, SS Ravi, Algorithmic aspects of topology control problems for ad hoc networks, in Proceedings of the 3rd ACM international symposium on Mobile Ad Hoc Networking and Computing (MobiHoc), Lausanne, Switzerland, pp. 123-134 (2002)

33. WT Chen, NF Huang, The strongly connecting problem on multihop packet radio networks. IEEE Trans Commun. 37(3), 293-295 (1989). doi:10.1109/ 26.20105

34. D Blough, M Leoncini, G Resta, P Santi, On the symmetric range assignment problem in wireless ad hoc networks, in Proc of the 2nd IFIP International Conference on Theoretical Computer Science (TCS), Deventer, The Netherlands, pp. 71-82 (2002)

35. LM Kirousis, E Kranakis, D Krizanc, A Pelc, Power consumption in packet radio net-works. Theor Comput Sci. 243(1-2), 289-305 (2000). doi:10.1016/ S0304-3975(98)00223-0

36. Y Wang, XY Li, Minimum power assignment in wireless ad hoc networks with spanner property. J Comb Optim. 11, 99-112 (2006). doi:10.1007/ s10878-006-5980-0

37. Y Zhu, M Huang, S Chen, Y Wang, Cooperative energy spanners: energyefficient topology control in cooperative ad hoc networks, in Proc of IEEE 30th Conference on Computer Communications (INFOCOM11), Miniconference, Shanghai, China, pp. 231-235 (2011)

38. M Bahramgiri, MT Hajiaghayi, VS Mirrokni, Fault-tolerant and 3-dimensional distributed topology control algorithms in wireless multi-hop networks, in Proceedings of the 11th Annual IEEE International Conference on Computer Communications and Networks (ICCCN), Miami, Florida, USA, pp. 392-397 (2002)

39. Y Wang, F Li, T Dahlberg, Power efficient 3-dimensional topology control for ad hoc and sensor networks, in Proc of IEEE Global Telecommunications Conference (GlobeCom 2006), San Francisco, CA, USA, pp. 1-5 (2006)

40. Y Wang, F Li, TA Dahlberg, Energy-efficient topology control for 3dimensional sensor networks. Int J Sensor Netw (IJSNet). 4(1/2), 68-78 (2008). doi:10.1504/IJSNET.2008.019253

41. Y Wang, L Cao, TA Dahlberg, F Li, X Shi, Self-organizing fault tolerant topology control in large-scale three-dimensional wireless networks. ACM Trans Auto Adap Syst (TAAS). 4(3), 19:1-19:21 (2009)

42. A Ghosh, Y Wang, B Krishnamachari, Efficient distributed topology control in 3-dimensional wireless networks, in Proc of 4th Annual IEEE Communications Society Conference on Sensor, Mesh and Ad Hoc Communications and Networks (SECON'07), San Diego, California, USA, pp. 91-100 (2007)

43. S Poduri, S Pattem, B Krishnamachari, GS Sukhatme, Using local geometry for tunable topology control in sensor networks. IEEE Trans Mob Comput. 8(2), 218-230 (2009) 
44. J Kim, J Shin, Y Kwon, Adaptive 3-dimensional topology control for wireless ad-hoc sensor networks. IEICE Trans on Communications. E93-B(11), 2901-2911 (2010). doi:10.1587/transcom.E93.B.2901

45. Y Zhang, L Cheng, A distributed protocol for multi-hop underwater robot positioning, in Proceedings of IEEE International Conference on Robotics and Biomimetics, Shenyang, China, pp. 480-484 (2004)

46. Z Zhou, JH Cui, S Zhou, Localization for large-scale underwater sensor networks, in Proceedings of IFIP Networking'07, Atlanta, GA, USA, pp. 108-119 (2007)

47. W Cheng, A Teymorian, L Ma, X Cheng, X Lu, X Lu, Underwater localization in sparse 3D acoustic sensor networks, in Proceedings of the 27th IEEE Conference on Computer Communications (INFOCOM'08), Phoenix, AZ, USA, pp. 236-240 (2008)

48. P Bose, P Morin, I Stojmenovic, J Urrutia, Routing with guaranteed delivery in ad hoc wireless networks, in Proc of 3rd Int Workshop on Discrete Algorithms and Methods for Mobile Computing and Communications (DIALM'99), Seattle, Washington, USA, pp. 48-55 (1999)

49. M Seddigh, JS Gonzalez, I Stojmenovic, RNG and internal node based broadcasting algorithms for wireless one-to-one networks. ACM Mob Comput Commun Rev. 5(2), 37-44 (2002)

50. B Karp, H Kung, GPSR: Greedy perimeter stateless routing for wireless networks, in Proc of the 6th ACM Annual International Conference on Mobile Computing and Net-working (MobiCom 2000), Boston, Massachusetts, pp. 243-254 (2000)

51. Y Wang, XY Li, Localized construction of bounded degree and planar spanner for wire-less ad hoc networks. ACM/Springer Mob Netw Appl (MONET). 11(2), 161-175 (2006). doi:10.1007/s11036-006-4469-5

52. WZ Song, Y Wang, XY Li, Localized algorithms for energy efficient topology in wireless ad hoc networks. ACM/Springer Mob Netw Appl (MONET). 10(6), 911-923 (2005). doi:10.1007/s11036-005-4447-3

53. XY Li, WZ Song, W Wang, A unified energy-efficient topology for unicast and broadcast, in Proceedings of the 11th ACM Annual International Conference on Mobile Computing and Networking (MobiCom'05), New York, USA, pp. 1-15 (2005)

54. XY Li, PJ Wan, Y Wang, CW Yi, O Frieder, Robust deployment and fault tolerant topology control for wireless ad hoc networks. Wiley J Wirel Commun Mob Comput. 4, 109-125 (2004). doi:10.1002/wcm.161

55. I Stojmenovic, Dominating set based bluetooth scatternet formation with localized maintenance, in Proceedings of the 16th International Parallel and Distributed Processing Symposium (IPDPS 2002), Washington, DC, USA, p. 122 (2002)

56. Y Wang, XY Li, O Frieder, Distributed spanner with bounded degree for wireless networks. Int J Found Comput Sci. 14(2), 183-200 (2003). doi:10.1142/S0129054103001674

57. S Rührup, C Schindelhauer, K Volbert, M Grünewald, Performance of distributed algorithms for topology control in wireless networks, in Proceedings of the 17th International Parallel and Distributed Processing Symposium (IPDPS 2003), Nice, France, (2003)

58. L Jia, R Rajaraman, C Scheideler, On local algorithms for topology control and routing in ad hoc networks, in Proceedings of the 15th Annual ACM Symposium on Parallel Algorithms and Architectures (SPAA 03), San Diego, California, USA, pp. 220-229 (2003)

59. C Schindelhauer, K Volbert, M Ziegler, Geometric spanners with applications in wireless networks. Comput Geom Theory Appl. 36, 197-214 (2007). doi:10.1016/j.comgeo.2006.02.001

doi:10.1186/1687-1499-2012-157

Cite this article as: Li et al:: Localized geometric topologies with bounded node degree for three-dimensional wireless sensor networks. EURASIP Journal on Wireless Communications and Networking 2012 2012:157.

\section{Submit your manuscript to a SpringerOpen ${ }^{\circ}$ journal and benefit from:}

- Convenient online submission

- Rigorous peer review

- Immediate publication on acceptance

- Open access: articles freely available online

- High visibility within the field

- Retaining the copyright to your article

Submit your next manuscript at $\gg$ springeropen.com 